\title{
Documentation of vascular risk factors in vascular dementia
}

\author{
Rahul Rao
}

\begin{abstract}
The diagnosis of vascular dementia may often be made on the basis of structural nouroimaging, this may not always be rellable. In view of this, a retrospective study of 30 case notes with diagnosis of vascular dementia was undertaken to examine the documentation of vascular risk factors and presence of cerebrovascular disease. Specific recommendations were then made for the future case note documentation. Nine months later, 10 case notes were examined to assess the impact of the recommendations. A noticeable improvement in the documentation of most risk factors was observed. The wider implications of these findings are discussed.
\end{abstract}

The definition of vascular dementia has undergone dramatic changes during the past 50 years. Stroke remains the most consistent risk factor for this disorder, and the currently accepted clinical guidelines for the diagnosis of vascular dementia according to classificatory systems such as DSM-IV (American Psychiatric Association, 1994) are synonymous with that of stroke. Although structural neuroimaging is a useful adjunct in the detection of vascular lesions, the temporal relationship between radiological evidence of infarction and cognitive decline is not always clear (Brust, 1988). As such, the documentation of risk factors for cerebrovascular disease (vascular risk factors) may aid the diagnostic process.

\section{The study}

In May 1996, case notes were obtained for patients aged over 65 on the Maudsley dementia case register $(n=15)$ and for in-patients admitted between April 1995 and March $1996(n=15)$, all patients having a primary ICD-10 diagnosis of vascular dementia. In each case, notes were examined for documentation of the following:

(a) The presence/absence of recent event(s) suggestive of cerebrovascular disease (e.g. neurological symptoms/acute deterioration in cognitive function).

(b) The presence/absence of the following risk factors: hypertension, diabetes mellitus, smoking, alcohol intake, history of arrhythmia and past history of transient ischaemic attack/stroke. (c) Physical examination for the following clinical signs: hypertension/hypotension, valvular disease, carotid bruits, retinopathy, gait abnormalities, and focal neurological signs (pyramidal/extrapyramidal/ cerebellar).

(d) That the following investigations had been requested: full blood count, plasma viscosity, blood glucose, serum cholesterol. syphilis serology (venereal disease research laboratory/Treponema pallidum haemagglutination test), chest X-ray, electrocardiogram and computerised tomography scan.

After the first stage of the audit loop, a letter was sent to all medical staff with modified recommendations for the future documentation of vascular risk factors in cases where a diagnosis of vascular dementia is suspected. The letter was sent out again a week after the change-over in junior medical staff. Nine months later, 10 case notes were examined for patients with a diagnosis of vascular dementia admitted during the nine-month period between May 1996 and January 1997, and the documentation of the modified recommendations re-examined.

\section{Findings}

First stage of the audit cycle

During the year from April 1995-March 1996, there were 16 finished consultant episodes for in-patients with a diagnosis of vascular dementia. One set of case notes could not be traced, the remaining 15 were included in the study. Thirty-five patients with vascular dementia were on the case register, but only 18 case notes could be traced, 15 of which were chosen at random for inclusion in the study. Results for the case note documentation of the above variables are shown in Table 1 . Serum cholesterol levels of more than $6.5 \mathrm{mmol} / 1$ were found in six of the 15 in-patients, but in none was any comment made in the clinical notes about these results. As admissions for 10 of the 30 case notes were not first assessments, the documentation of the presence/absence of symptoms 
Table 1. Vascular risk factor documentation in primary and secondary phases of audit

\begin{tabular}{|c|c|c|}
\hline Risk factor documented & $\begin{array}{l}n(\%) \\
\text { (First } \\
\text { phase) }\end{array}$ & $\begin{array}{l}n(\%) \\
\text { (Second } \\
\text { phase) }\end{array}$ \\
\hline History of hypertension & $11(37)$ & $6(60)$ \\
\hline History of diabetes mellitus & $2(7)$ & $4(40)$ \\
\hline Smoking & 15 (5) & $10(100)$ \\
\hline Alcohol intake & $15(50)$ & $10(100)$ \\
\hline History of arrhythmia & $13(43)$ & $4(40)$ \\
\hline $\begin{array}{l}\text { History of transient ischaemic } \\
\text { attack/stroke }\end{array}$ & $13(43)$ & $6(60)$ \\
\hline Examination of arrhythmia & $26(87)$ & $6(60)$ \\
\hline Blood pressure measurement & $27(90)$ & $10(100)$ \\
\hline Heart sounds (murmurs) & $23(77)$ & $10(100)$ \\
\hline Carotid bruits & $9(30)$ & $6(60)$ \\
\hline Examination of fundi & $18(60)$ & $8(80)$ \\
\hline Gait abnormalities & $14(47)$ & $4(40)$ \\
\hline Pyramidal signs & $23(77)$ & $8(80)$ \\
\hline Other focal signs & 7 (23) & $4(40)$ \\
\hline Full blood count & $27(90)$ & $10(100)$ \\
\hline Plasma viscosity & $26(87)$ & $8(80)$ \\
\hline Blood glucose & $26(87)$ & $10(100)$ \\
\hline Serum cholesterol & $16(53)^{1}$ & $10(100)$ \\
\hline Syphilis serology & $24(80)$ & $8(80)$ \\
\hline Chest X-ray & $18(60)$ & $8(80)$ \\
\hline Electrocardiogram & $14(47)$ & $8(80)$ \\
\hline CT scan & $25(83)$ & $10(100)$ \\
\hline
\end{tabular}

1. Serum cholesterol $>6.5 \mathrm{mmol} / \mathrm{l}$ in six cases.

suggestive of cerebrovascular disease was not considered valid for inclusion in criteria to be laid down for the second stage of audit. The other modification for future documentation was that a CT scan was not considered necessary if this had been carried out during a previous assessment, unless there was clear evidence from the notes that there had been an acute cognitive decline.

\section{Completing the audit loop}

Thirteen finished consultant episodes were recorded between May 1996 and January 1997. All case notes were traced and 10 selected at random. None of these case notes had been examined during the first part of the audit loop, the results of the re-audit are also shown in Table 1. Documentation of most of the recommended criteria were higher in the second stage of case note examination. The exceptions to this were a history of and examination for arrhythmia and examination of gait.

\section{Comment}

Vascular dementia remains a potentially treatable disorder, particularly in terms of secondary prevention (Hachinski, 1992). As stroke shows the most consistent association with this dementia, this study was confined to risk factors known to be associated with stroke (Drachman, 1993). During the first phase of the audit loop, there was a lack of historical information relating to risk factors for cardiovascular disease in more than $50 \%$ of cases. On physical examination, there was no documentation of either gait or fundal examination in $40 \%$ or more of cases. This is relevant, as changes in gait are known to be more common in vascular dementia than in Alzheimer's disease (Kotsoris et al, 1987). Seventy per cent had no record of examination for carotid bruits and $53 \%$ had no electrocardiogram performed, which is striking in view of carotid stenosis and arrhythmia both being strongly associated with vascular dementia and potentially remediable (Skoog, 1994). The other notable finding was of hypercholesterolaemia in nearly half the in-patient sample, especially as serum cholesterol had not been performed in $47 \%$ of all cases. The results of the second phase are encouraging, with a case note documentation of $100 \%$ for important risk factors such as smoking/alcohol intake, blood pressure and blood glucose/serum cholesterol.

The findings from this study are relevant in two respects. First, with the rising elderly population and the frequent requests from general practitioners for the assessment of cognitive impairment, the role of psychiatrists in the diagnosis of vascular dementia should not be understated. Second, it is known that the presence of vascular lesions seen on computerised tomography does not per se indicate causality, particularly where the onset of dementia occurs several weeks or months after a stroke (Lopez et al, 1994). For example, in one longitudinal study of dementia occurring three months after stroke, the development of dementia at this stage was attributed to stroke in only $26 \%$ of cases (Tatemichi et al, 1993). Therefore overemphasis on computerised tomography findings may be misleading and the documentation of recognised vascular risk factors may improve the reliability of the diagnostic process. The documentation of vascular risk factors also has implications for both the prevention of cognitive decline where treatable risk factors (such as hypertension) are discovered and for use in epidemiological studies of dementia. The use of larger projects may seek to clarify these issues.

\section{Acknowledgements}

I thank Mr John Harris for his help with tracing case notes, Dr Clive Holmes for access to case register information and Dr Marisa Silverman for her help and encouragement during the project. 


\section{References}

AMERICAN PSYChIATRIC ASSOCLATION (1994) Diagnostic and Statistical Manual of Mental Disorders (4th edn) (DSMIV). Washington, DC: APA

BRUST, J. C. (1988) Vascular dementia is overdiagnosed. Archives of Neurology, 46, 799-801.

DRACHMAN, D. A. (1993) New criteria for the diagnosis of vascular dementia: do we know enough yet? Neurology. 43. 243-245.

HACHINSK, V. C. (1992) Preventable senility: a call for action against vascular dementias. Lancet, 340, 645-648.

KOTSORIS, H., BARCLAY, L. L., KHEYFETS, S., et al (1987) Urinary and gait disturbances as markers for early multi-infarct dementia. Stroke, 18, 138-141.
LOPEZ, O. L. LARUMBE, M. R. BECKER, J. T., et al (1994) Reliability of the NINDS-AIREN clinical criterla for the diagnosis of vascular dementia. Neurology. 42, 12401245.

SкооG, I. (1994) Risk factors for vascular dementia: a review. Dementia, 5, 137-144.

TATEMichi, T. K., Desmond, D. W.. PAiK, M., et al (1993) Clinical determinants of dementia related to stroke. Annals of Neurology. 33, 568-575.

Rahul Rao, Portnalls Unit, Farnborough Hospital, Farnborough Common, Orpington, Kent BR6 8ND

\section{తิ Unwillingly to School}

Fourth edition

\section{Edited by lan Berg and Jean Nursten}

This book describes the epidemiological aspects of school absence and offers practical help to those who are faced with helping children who fail to attend school.A multidisciplinary approach to truancy and school refusal is put forward, drawing on experience from the UK, the United States, Sweden and New Zealand. The clinical features of the various underlying conditions are also demonstrated, and the future prospects of those who display this problem outlined. This new edition of a well respected book has been comprehensively rewritten to take into account current research and thinking. £20.00, 336pp., 1996, ISBN 0902241893

Available from bookshops and from the Publications Department, Royal College of Psychiatrists, 17 Belgrave Square, London SW1X 8PG (Tel. 0171-235 2351, extension 146)

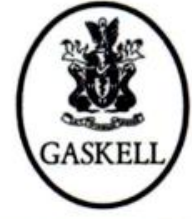

